\title{
IN SITU MICROASSEMBLY
}

\section{Ronaldo Ronaldo, Thomas Papastathis, Hongyi Yang, Carsten Tietje, Michele Turitto, Svetan Ratchev}

The University of Nottingham, Precision Manufacturing Centre,

School of Mechanical, Materials and Manufacturing Engineering,

Nottingham, NG7 2RD, UK

\begin{abstract}
The 21 st century sees significant breakthroughs in fabricating micro devices in the quest of miniaturising. Most micro parts have been manufactured in the range of less than $1 \mathrm{~mm}$. However, they are built based on material that is process dependant, resulting in monolithic parts. For example the Integrated Circuits, Micro Electro Mechanical System (MEMS) are silicon based, and on their own do not constitute a complex system that requires various functions. To pursue fully functional and miniaturised complex devices, microassembly is therefore necessary. However, microassembly processes differ from the assembly processes in the macro world. Microassembly encounters sticking effects in parts handling, adhesive forces from electrostatic attraction, van der Walls forces and surface tension $[1,2]$. This paper envisions microassembly processes by using an innovative approach. It departs from the traditional assembly process by utilising the Projection Micro Stero Lithography, with a positioning algorithm to assemble micro parts without traditional handling and joining, named in situ microassembly process.
\end{abstract}

Keywords microassembly, in situ microassembly, projection micro stereolithography, rapid prototyping.

\section{Problems in Microassembly}

Micro products are defined as products whose functional features or at least one dimension are in the order of micrometers. The products are usually characterised by a high degree of integration of functionalities and components [3].

The continuous miniaturisation of parts has led to difficulties in handling, transporting, gripping and positioning whilst assembling them. Furthermore, these difficulties have naturally led to optimisation in vision, precision positioning and miniaturisation of assembling tools. Vision systems help the assembly process, however the limitations lie on the short depth of focus which creates difficulties during the manipulation process [2]. For the assembly of micro components, this restriction is a major barrier especially when two micro parts are required to be monitored during the whole process. 
Miniaturisation of assembly tools, such as micro grippers, has adverse effects associated with contact to micro parts, such as the electrostatic attraction, and van der Walls forces, and contamination. These effects have been avoided by a number of ways of non contact handling utilising magnetic, electric, optical, aerodynamic, ultrasonic methods, as reviewed by Vandaele (2005) [4], or by pneumatics as used for a contactless feeder reported in [5].

Böhringer et al (1998) having studied extensively the difficulties of assembling micro parts serially, envisioned the idea for parallel microassembly and introduced the self assembly concept based on electrostatic force fields as a solution [1]. Despite many successful publications on self-assembly, the assembly process of single parts providing true three dimensional structures should not be ignored. Thus, a direct control and in situ microassembly with the utilisation of photo-projection micro stereolithography is one of the solutions for non self-assembled material. The system can be extended further with contactless feeders and handling systems, to form a holistic approach encompassing the whole production cycle.

\section{In situ assembly methodology}

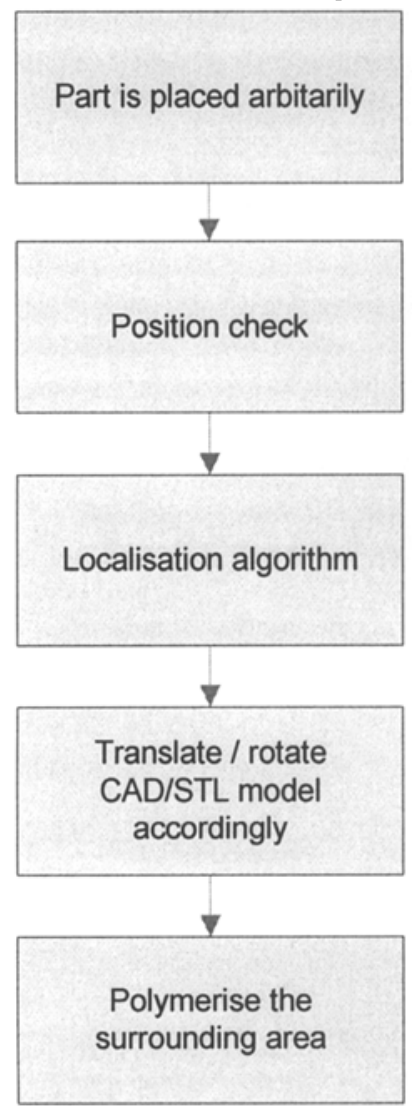

The part to be assembled can be transported with an ordinary vibratory feeder, ultrasonic plate feeder, or contactless feeder. The part is placed with reasonable positioning accuracy on the working table.

A small amount of liquid photopolymer is then added to surround the part. The amount of the liquid polymer must be at least sufficient for a layer of polymerisation. The part must be on its equilibrium state while resting on the working table.

The vision system takes measurements of some points on the parts. These points serve as datum points that will be used later for the localisation algorithm.

Once the positioning of the part is detected, the algorithm, implemented in software, calculates the Euclidean displacement of the part in comparison to the part of the model file. Euclidean displacement and transformation are explained in detail in the workpiece localisation algorithm section.

As long as the part is located within reasonable angle, the Computer Aided Design (CAD) or Stereolithography (STL) model can be translated and rotated as much as the Euclidean displacement to match the placement orientation of the part. The new

CAD or STL model orientation and location must be within the built area of the polymerisation process, so the whole product can be built successfully. 
Finally, the polymer around the part is solidified by photo projection. For further parts to be assembled, a similar approach can be taken. If it is not possible, then the outline around the part can be solidified to provide the exact imprint for the part to be placed in the correct orientation and place. Once it is placed, the solidifying process can take place layer by layer by the Projection Micro Stereo Lithography process until the whole product is built. This process is described in the following section.

\subsection{Projection Micro Stereo Lithography}

Projection Micro Stero Lithography $(\mathrm{P} \mu \mathrm{SL})$ - utilises a UV light source and a digital micro mirror display to project and solidify a layer at once. 3D microstructures with $120 \mathrm{~nm}$ resolutions are reported in [6].

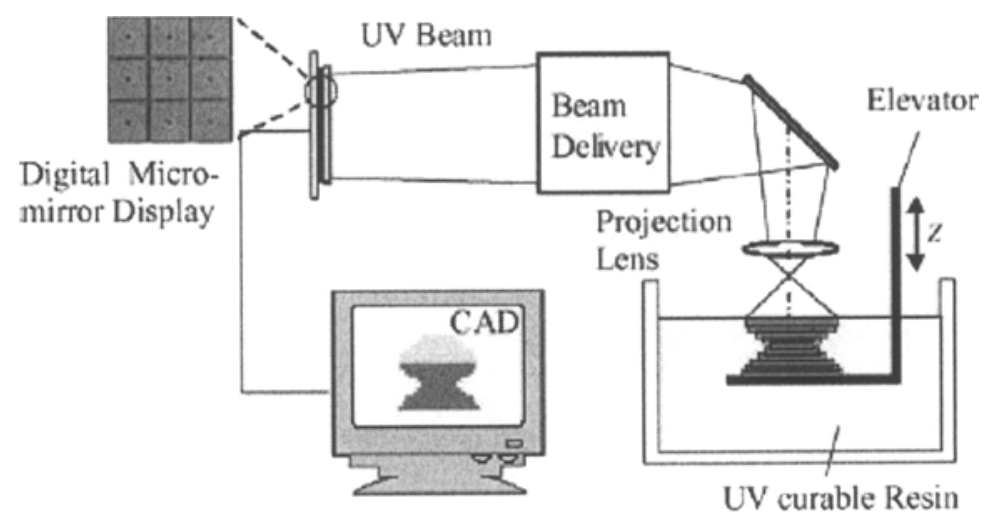

Fig. 1. Schematic diagram of $\mathrm{P} \mu \mathrm{SL}$ using digital micro mirror display [7]

Fig. 1 shows the principle of of $\mathrm{P} \mu \mathrm{SL}$ in a schematic way. The system contains a state of the art product Digital Light Processor (DLP $\left.{ }^{\infty}\right)$ from Texas Instruments. The Digital Light Processor $\left(\right.$ DLP $\left.^{\infty}\right)$ contains a 1.3 million Digital Micromirror Device (DMD) and can reflect 1024 shades of gray to create a grayscale image. When the micromirror is tilted towards the photopolymer liquid, it projects a photon that is absorbed by the photo-initiator and generates radicals. These radicals then react with the rest of the monomers until a stable polymer chain is formed. These chains lead to the solidification of the polymer [7].

The advantage of this concept in comparison to the more commonly known stereolithography process is that in P $\mu \mathrm{SL}$ the polymerisation across the whole surface of the layer is done at once due to the planar projection. Whereas the ordinary stereolithography has a laser beam that traces a cross section pattern on the surface of the photopolymer. Therefore P $\mu \mathrm{SL}$ is faster and for the case of in situ microassembly process, $\mathrm{P} \mu \mathrm{SL}$ is superior due to the capability to polymerise the surrounding area of the part at once, and hence a more accurate geometry can be achieved. 
As with any other Rapid Prototyping System, P $\mu$ SL has no measurement and feedback to control the process.

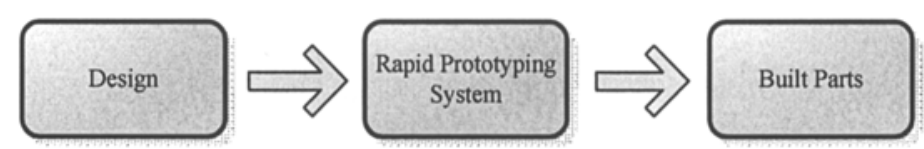

Fig. 2. Urdınary Kapıd Prototyping System

\subsection{Measurement system}

In in situ microassembly, a measurement system is used to define the position of the parts to be assembled on the working table. A non contact measurement technique is preferred as it does not exert any force onto the object, and hence the part will always stay in its equilibrium state. Non contact measurement techniques such as laser interferometry, ultrasound or optical measurement can be utilised. Fraunhofer IPT Germany reported utilising the Digital Micromirror Device for lateral scanning with resolution of $1 \mu \mathrm{m}$ in 2001[8]. The schematic diagram below is proprietary of GFMesstechnik (patent no. EP0943950A 1).

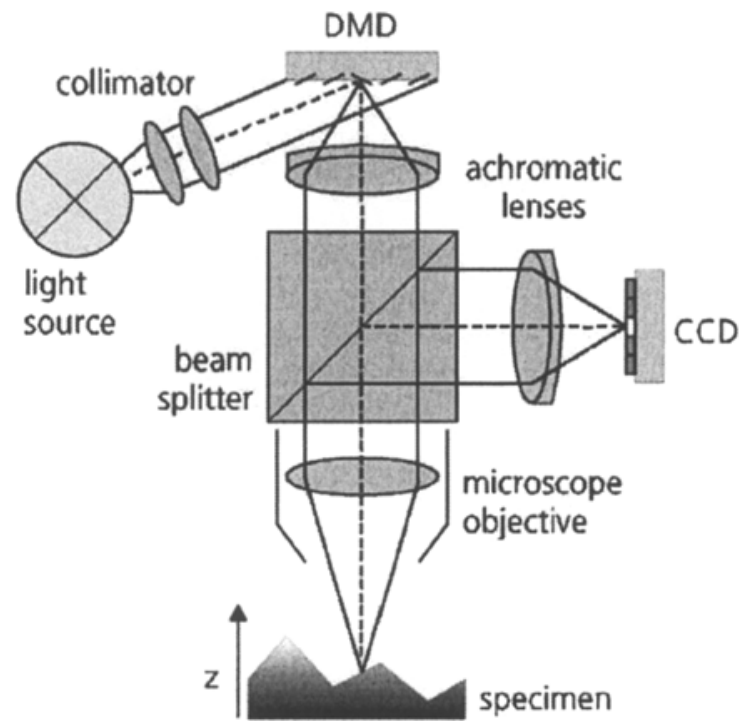

Fig. 3. Schematic diagram of optical metrology using DMD [8]

Integrating the $\mathrm{P} \mu \mathrm{SL}$ and the optical metrology, both utilising the same DMD technology, is the most desired solution. Because the whole system uses the same 
DMD component there is no need to alter the structure of the P $\mu$ SL system, and therefore representing the most cost effective solution.

In terms of precision, utilising the DMD as a metrology and fabrication tool will be the most precise solution for the reason that DMD is fixed to the same location and both processes are conducted by the same device, and therefore there is no device performance discrepancy.

For the in situ microassembly, the measurement system is only used to check the position of parts that are going to be assembled, as the light source can polymerise the uncured polymer and alter the intended geometry.

\section{Workpiece Localisation Algorithm}

Workpiece localisation algorithms have been researched in depth and applied widely for positioning correction in conventional machining. The typical examples of such algorithms include the variational algorithm, tangent algorithm and Menq algorithm. In particular, the localisation of 3D objects has been developed by Menq, Yau and Lai (1992) as a least squares problem with non linear optimisation. HongTan algorithm is a similar iterative technique where the home surface point and the Euclidean transformation are optimised separately. The computational time based on Hong-Tan algorithm with 45 measurement points using a Pentium PC (166MHz) is reported to be under one second [9].

The functional model that represents the 3D template and surface discretely by the least square matching concept had been developed in parallel by Gruen (1984), Ackermann (1984) and Pertl (1984) [10]. The basic model is shown below:

$$
f(x, y, z)=g(x, y, z)
$$

Where the $f(x, y, z)$ is template model location and $g(x, y, z)$ is the search element location. However the ideal equation above is not true in the real world, as it contains random or stochastic error. The true error vector $e(x, y, z)$ is noted as with the equation below:

$$
f(x, y, z)-e(x, y, z)=g(x, y, z)
$$

Nevertheless, the aim is always to minimise the error and to converge the search element location as closest to the template model location through iterative process. In conventional machining operations that utilise the workpiece localisation algorithm, it is required to move the object from the search element location to the template model location. The process is represented by transformation matrices and is called Euclidean transformation). 


\subsection{Application of the localisation algorithm to in situ microassembly}

The localisation algorithm can be applied in the in situ microassembly for an accurate assembly process. The process starts by placing a part to be assembled on the working table. Once it is placed, an optical measurement is used to obtain the actual part position.

Applying the localisation algorithm, which compares the datum points of the CAD model with the actual measurement, gives the Euclidean distances. This is the distance between the template model position and the actual part position.

Once the Euclidean distance is acquired, the next action is to perform the Euclidean transformation, that is to translate and rotate either the template model or the part to closest match into the ideal model $f(x, y, z)=g(x, y, z)$.

When performing the physical translation of the part, the actual position and the intended one will not match completely. This is caused by both the positional error of the stage used to re-position the part and the relative displacement of part to the stage surface, since the part is not held rigidly by a fixturing device.

A more accurate concept is introduced in the in situ microassembly process. The positional correction is done through feeding the error values to translate the $\mathrm{CAD} / \mathrm{STL}$ model. This concept is far more accurate due to the fact that no physical movement is experienced by the parts, and hence the eliminating the transport, handling and orientation errors are eliminated.

Once the CAD/STL model is translated to the match the position of the part, the 'updated' model is then sent to the P $\mu \mathrm{SL}$ system. The surrounding medium of the part is solidified and it is then securely held. The process may be repeated to assemble different parts on different layers.

To summarise, there are four components in the in situ microassembly process, the design, localisation algorithm, the P $\mu \mathrm{SL}$, and measurement system. These components work mutually to build complex and functional devices.

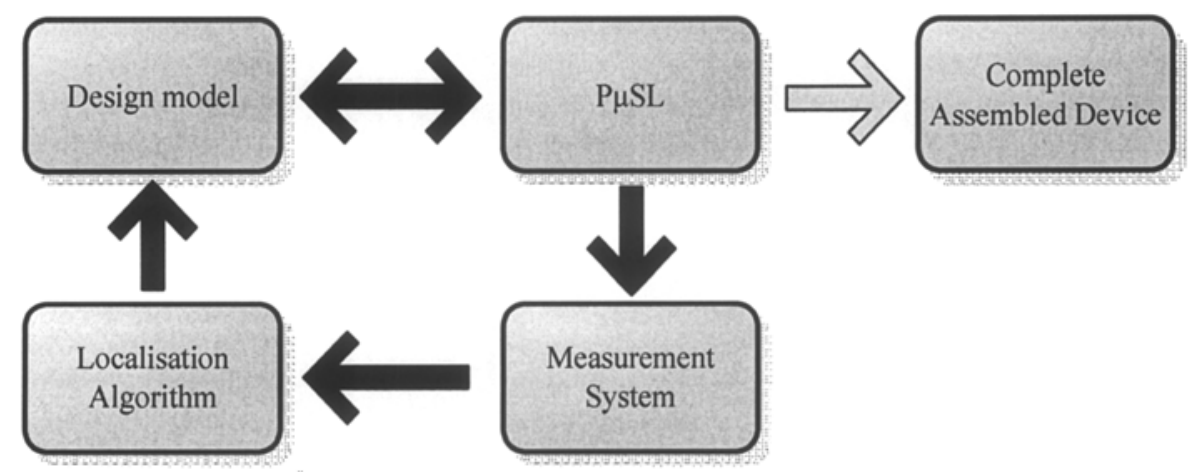

Fig. 4. In Situ Microassembly components

An experimental study has been carried out to validate the concept and applicability of in situ microassembly process. This case study is described in the following section. 


\subsection{Case Study}

A battery and a Light Emitting Diode (LED) are assembled as a mini torch. The width and height of the conductive LED legs are $480 \times 480 \mu \mathrm{m}$ and the battery has a diameter of $7.7 \mathrm{~mm}$ and depth of $5.3 \mathrm{~mm}$ (Figure 5 ). The mini torch is designed as the model below (Figure 6).

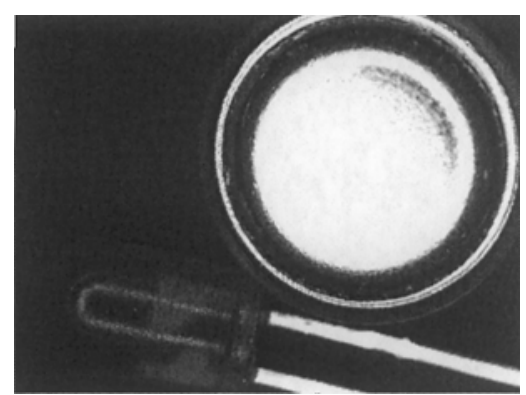

Fig. 5. A battery and an LED

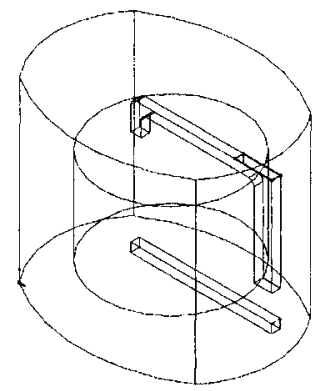

Fig. 6. The CAD model

If the design were to be manufactured conventionally, the casing would have to be split into two as it is not possible to machine the battery cavity and LED leg channels from a single part. Therefore the final assembly process constitutes of handling, orientation, and joining of four parts; the first half case, battery, LED and the other half case to make the final product.

In the case of in situ microassembly, the casing with the complex cavity and micro channels are built around the parts layer by layer. Firstly, the CAD model is converted to STL format. The LED is placed on the working table. To validate the concept, the LED is placed at the angle of 27 degree. The STL model is then rotated by 27 degrees to accommodate the error. The new STL model is then transferred to the P $\mu$ SL system (Perfactory). Fig. 7 shows the STL model and the rotation process. Stereolithography process then takes place solidifying the polymer around the LED and battery according to the new orientation of the STL model.

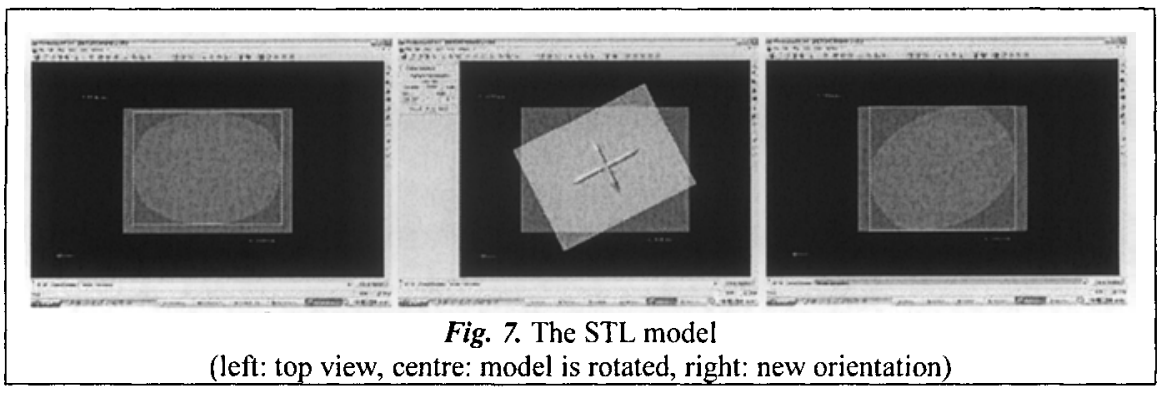


The mini torch has been successfully fabricated through layer by layer solidification of polymer with the LED and the battery was being assembled through the in situ microassembly process as shown by the figures below.

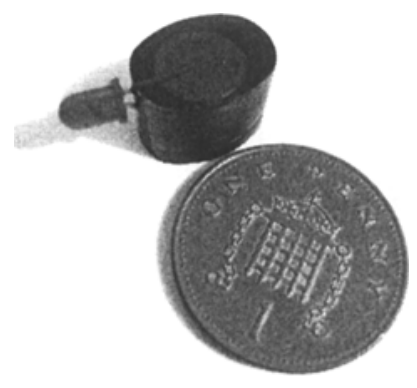

Fig. 8. In situ micro assembled mini torch

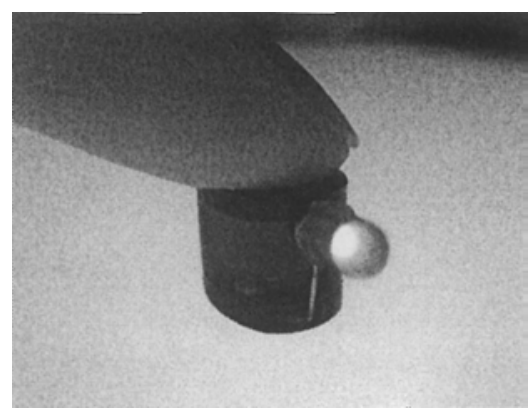

Fig. 9. Fully functional mini torch

\section{Conclusions and outlook}

The case study has confirmed the applicability of the in situ microassembly process that could be used instead of the conventional microassembly processes, and the same principle can be applied to even smaller devices. The design rule of thumb is to place the largest surface area of the part away from the projection path of the Stereolithography beam to ensure that no geometry of the part blocks the projection of the beam.

Accuracy of the in situ microassembly process depends on the accuracy of the measurement system. Depending on the need, different measurement systems can be used. However, the DMD measurement system is a promising system that can be integrated with the $\mathrm{P} \mu \mathrm{SL}$. The local minimisation algorithm can also be integrated into the design software. Once all the components are integrated together, in situ microassembly process can take place autonomously, being a solution for the creation of complete and functional micro devices. For small quantity production, in situ microassembly process is the most cost effective as it does not require any expensive tooling.

\section{References}

1. Böhringer, K.F., R.S. Fearing, and K.Y. Goldberg, The Handbook of Industrial Robotics: Microassembly. 2nd ed. 1999: John Wiley \& Sons.

2. Van Brussel, H., et al., Assembly of microsystems, in Cirp Annals 2000: Manufacturing Technology, Vol 49/2/2000. 2000. p. 451-472.

3. Alting, L., et al. Micro Engineering. in CIRP. 2003. 
4. Vandaele, V., P. Lambert, and A. Delchambre, Non-contact handling in microassembly: Acoustical levitation. Precision Engineering, 2005. 29(4): p. 491.

5. Turitto, M., Y.-A. Chapius, and S. Ratchev. Pneumatic Contactless Feeder for Microassembly. in International Precision Assembly Seminar. 2006. Bad Hofgastein, Austria: Springer.

6. Cox, A., C. Xia, and N. Fang, Microstereolithography: A Review. Proceeding of the 1st International Conference on Micromanufacturing, 2006.

7. Sun, C., et al., Projection micro-stereolithography using digital micro-mirror dynamic mask. Sensors and Actuators A: Physical, 2005. 121(1): p. 113.

8. Bitte, F., G. Dussler, and T. Pfeifer, 3D micro-inspection goes DMD. Optics and Lasers in Engineering, 2001. 36(2): p. 155.

9. Chu, Y.X., J.B. Gou, and Z.X. Li, Workpiece localization algorithms: Performance evaluation and reliability analysis. Journal of Manufacturing Systems, 1999. 18(2): p. 113.

10. Gruen, A. and D. Akca, Least squares $3 D$ surface and curve matching. ISPRS Journal of Photogrammetry and Remote Sensing, 2005. 59(3): p. 151. 